\title{
High serum non-high-density lipoprotein and the risk of ischemic stroke: A systematic review
}

\author{
Nyoman Tyas Apsari', Kumara Tini1,2, I Putu Eka Widyadharma ${ }^{1,2}$ \\ ${ }^{1}$ Faculty of Medicine, Udayana University, Bali, Indonesia \\ ${ }^{2}$ Department of Neurology, Sanglah Hospital, Bali, Indonesia
}

\begin{abstract}
Objectives. High levels of non high density lipoprotein cholesterol (non-HDL-C) are known to contribute to an increased risk of ischemic stroke. This systematic review was conducted to identify the relationship between high non-HDL-C levels and the risk of ischemic stroke.

Material and methods. This systematic review using PRISMA (preferred reporting items for systematic reviews and meta-analyses) statement guideline, with a pre-determined search strategy. The search was conducted in Pubmed Central (PMC) and Pubmed from 2009 until 2020 with inclusion criteria, patients with a diagnosis of ischemic stroke, age $>18$ years, with non-HDL-C levels. The title and abstract of these articles were reviewed for relevance, based on inclusion criteria. This systematic review using STROBE to evaluate the quality in individual study which consists of 22 domains.

Results. In the final stage, this systematic review identifies six cohort study consisting 166.720 participants. Overall, the quality in individual studies based on STROBE is good. We reported that there are two studies report that high non-HDL-C levels can significantly increase the risk of ischemic stroke. Meanwhile, there are four studies that report there was no significant relationship between high non-HDL-C levels and the risk of ischemic stroke.

Conclusions. Overall, this systematic review provides the result about relationship between high levels of nonHDL-C and the risk of ischemic stroke, there are two studies with a larger population support that high levels of non-HDL-C can increase the risk of ischemic stroke. However, further studies is needed with a large population that specifically identify the relationship between non-HDL-C levels and risk of ischemic stroke and makes a better adjustments for confounding variables.
\end{abstract}

Keywords: ischemic stroke, non-HDL-C, risk

\section{INTRODUCTION}

Globally, stroke places second as the cause of mortality and third as the cause of disability (1). Out of all stroke cases in the world, ischemic stroke accounts for $87 \%$ of cases, and $13 \%$ of them were hemorrhagic stroke. To reduce the prevalence of stroke, we need to identify risk factors and show success from efforts in reducing modified risk factors. These are important as a strategic intervention to reduce the risk of stroke. One of the risk factors that can be modified is dyslipidemia (2).

Dyslipidemia is marked by an increase in plasma cholesterol, triglycerides (TG), or both, or an increase in low-density lipoprotein-cholesterol
(LDL-C) and a decrease in high-density lipoprotein-cholesterol (HDL-C). These contribute to the development of atherosclerosis. Other than LDL-C and HDL-C, a new risk factor with the potential to be modified is non-high-density lipoprotein cholesterol (non-HDL-C). Non-HDL cholesterol is measured by the total plasma cholesterol subtracted by HDL cholesterol. Non-HDL cholesterol has a more comprehensive size from atherogenic lipoprotein compared to LDL only (3).

Non-HDL-C does not only contain LDL-C, but also cholesterol contained in all other apolipoprotein B (apoB), which contains potential atherogenic lipoprotein in blood, including cholesterol in lipo- 
protein a, intermediate-density lipoprotein, very low-density lipoprotein, and cholesterol-enriched residual lipoprotein. Experimental evidence supported a more important role of apoB and lipoprotein containing apoB compared to LDL-C in the process of atherogenesis. Measuring apoB and non-HDL-C is known to be highly correlated in several studies because TC and HDL-C are significantly affected by dietary intake. Meanwhile, non-HDL-C can be measured not only during fasting but also in a post-prandial state (4).

Based on the statement above, the author would like to understand the correlation between high serum level of non-high-density lipoprotein cholesterol (non-HDL-C) and the risk of ischemic stroke by conducting a systematic review of related studies.

\section{MATERIALS AND METHODS}

\section{Search strategy}

Article search was conducted from 2 databases, i.e. Pubmed Central (PMC) and Pubmed. It is conducted since the relevant articles were first published until 1 November 2020. A literature search was performed based on PRISMA system (preferred reporting items for systematic reviews and meta-analysis). While searching for articles or journals, the author used the following combination of keywords: ischemic stroke and non-high-density lipoprotein cholesterol OR nonHDL-C and risks [MeSH terms].

\section{Eligibility criteria}

A structured literature search was conducted to identify studies concerning the correlation between non-HDL-C serum level and the risk of ischemic stroke. The author used PICOS (population, intervention, comparison, outcome, and study design) in searching for articles, with the following inclusion criteria: 1) the population criterion includes patients diagnosed with ischemic stroke and aged more than 18 years old, 2) the intervention/exposure criterion includes patients with high serum level of non-high-density lipoprotein, 3) The comparison criterion includes patients with low serum level of non-high-density lipoprotein, 4) hazard ratio (HR) and $95 \%$ confidence interval $(95 \% \mathrm{CI})$ as outcomes, 5) a cohort study, and 6) studies using English.

\section{Data extraction}

Data were extracted by inputting relevant data from chosen studies manually and independently to a table. All process of data collection was conducted by the first author. All disagreements were resolved through a consensus.

\section{Quality assessment}

The author used the STROBE (strengthening the reporting of observational studies in epidemiology) checklist to evaluate observational studies. The quality of all parts was assessed as complete, less complete, and incomplete, each represented with the color green, yellow, and red.

\section{RESULTS}

\section{Study selection}

The search strategy identified 460 studies from 2 databases, with the details of 194 studies from Pubmed, and 266 studies from PMC. Afterward, duplicates were searched and deleted using the EndNote X9 application, resulted in 448 articles in which the title and abstract were to be reviewed according to the relevance to the theme of this systematic review. After screening, there were 14 articles to be reviewed based on the overall article. Then, 6 articles were used in this systematic review. The result of article selection was depicted in a 2009 PRISMA flowchart in Figure 1.

\section{Quality assessment}

This systematic review used the STROBE checklist to evaluate the quality of individual studies. In general, the quality of studies in this systematic review was considered good, showed by the dominating green color. The summary of quality assessment is presented in Table 1.

\section{Study characteristics}

This systematic review identified six cohort studies collected from four countries. Two studies were from the United States $(5,6)$, three were from Japan $(7,8,9)$, and one from China (10). The participants of all studies were patients aged more than 18 years old. The samples from each study vary, with a total of 166,720 participants. They consisted of 103,989 male participants and 62,731 female 


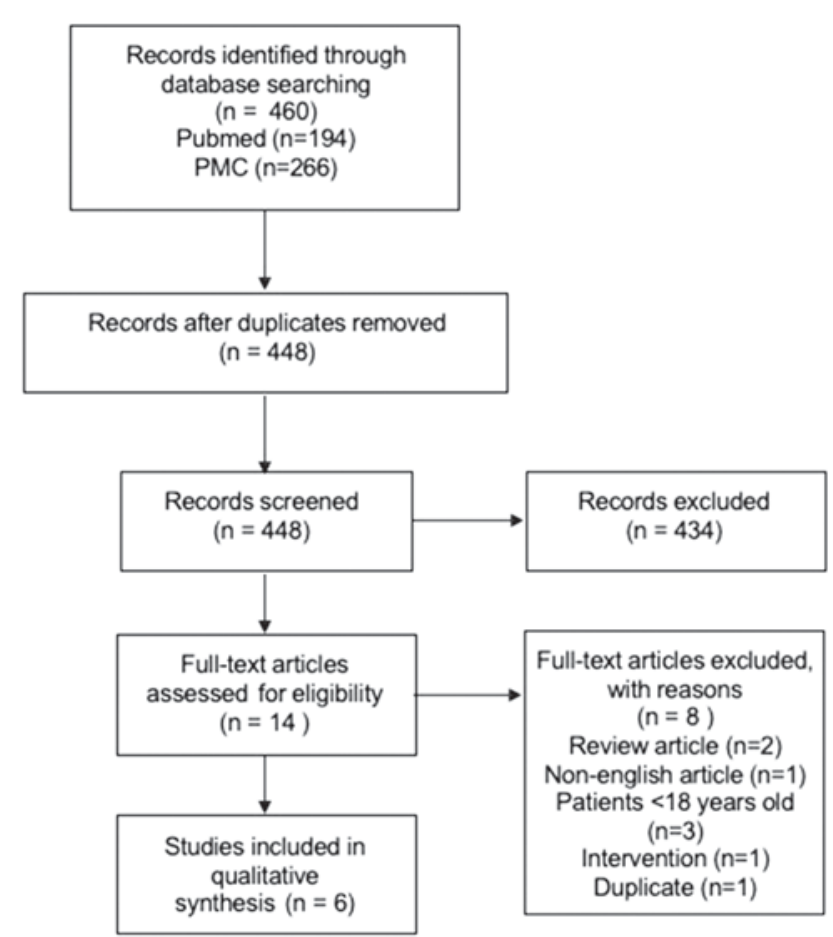

FIGURE 1. Flow diagram of study selection process

participants. All studies were published in 20092020. Each study had a population characteristic, follow-up duration, and varied threshold serum level of non-HDL-C. The summary of the studies' characteristics in this systematic review is presented in Table 2.

\section{Study results}

The author found six studies with results concerning the correlation between high serum level of non-HDL-C and the risk of ischemic strong using HR scale and $95 \%$ CI. Out of six studies, two of them showed that high serum levels of nonHDL-C significantly increased the risk of ischemic stroke $(5,10)$. Meanwhile, the other four studies showed an insignificant correlation between high serum level of non-HDL-C and the risk of ischemic stroke (6-9). More detailed study results can be seen in Table 2.

\section{DISCUSSION}

This systematic review summarized and analyzed six cohort studies, in which two studies were conducted in the United States $(5,6)$, three studies from Japan $(7,8,9)$ and one from China $(10)$. The total participants from all studies were 166,720 people, consisted of 103,989 male participants, and 62,731 female participants. Overall, the studies included in this systematic review had a good quality. The author found six cohort studies that investigated the correlation between high serum level of non-HDL-C and the risk of ischemic stroke, with the outcome of HR and 95\% CI. Two studies showed a significant correlation between high serum level of non-HDL-C and the risk of ischemic stroke $(5,10)$. Meanwhile, the other four studies showed an insignificant correlation between high serum level of non-HDL-C and the risk of ischemic stroke (6-9).

The results difference from these six journals can be due to various possibilities. The first difference was the inclusion and exclusion criteria of population characteristics. Studies with significant results included participants free from a stroke from the beginning to reduce the role of a confounding factor, which was the use of statin (5). The same was conducted in other studies with significant results, whereas it excluded participants who received cholesterol-lowering drugs (10). In three studies that showed insignificant results, the use of cholesterol-lowering drugs was only controlled by adjustment $(6,7,9)$, and one study did not observe and adjust the use of cholesterol-lowering drugs (8).

Regarding the sample size, studies with significant results had a larger sample size compared to other studies with insignificant results. In a larger sample size, the confidence interval $(95 \% \mathrm{CI})$ is narrowed, which showed better precision (11).

Other things that can affect result differences are other risk factors which can be a confounding variable. Other than hyperlipidemia, there are many risk factors for ischemic stroke. Risk factors that cannot be modified include age, gender, genetic, and race/ethnicity. Meanwhile, risk factors that can be modified include hypertension, smoking habit, waist-hip ratio, diet, physical activity, diabetes mellitus, alcohol consumption, heart disease, and apolipoprotein B (2). Adjustments to these confounding variables may lead to insignificant results.

A study in the United States consisted of $42 \%$ African Americans (AAs) and 58\% white race and found a significant result (5). This may be affected by the African Americans, who have twice the risk of having a stroke. One of the reasons for the correlation between race differences was the higher prevalence of the risk factors of stroke, such as hy- 


\begin{tabular}{|c|c|c|c|c|c|c|}
\hline 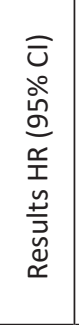 & 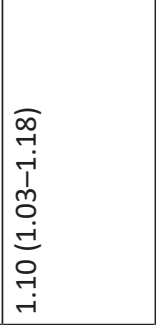 & 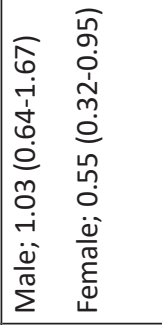 & 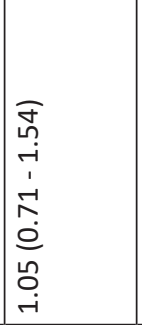 & 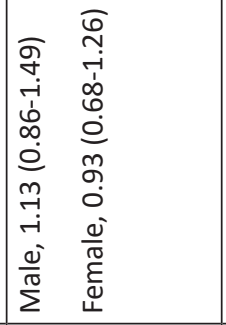 & 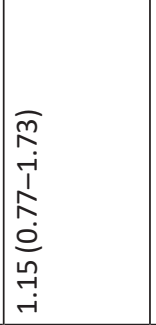 & 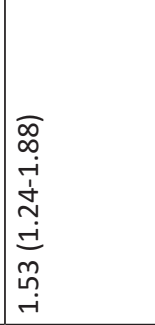 \\
\hline 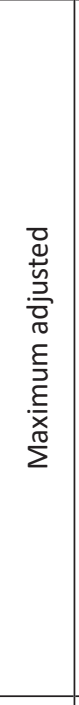 & 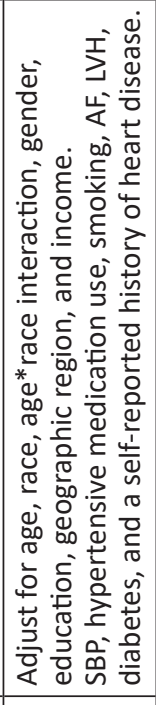 & 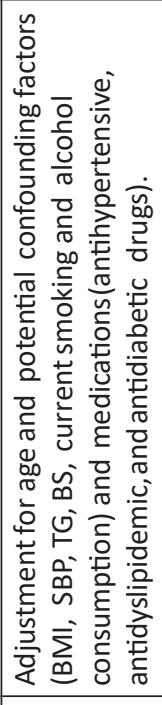 & 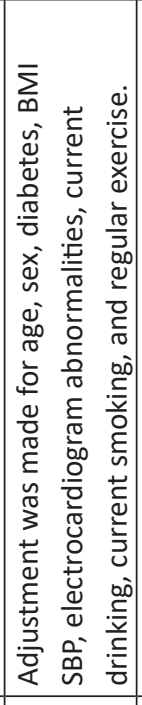 & 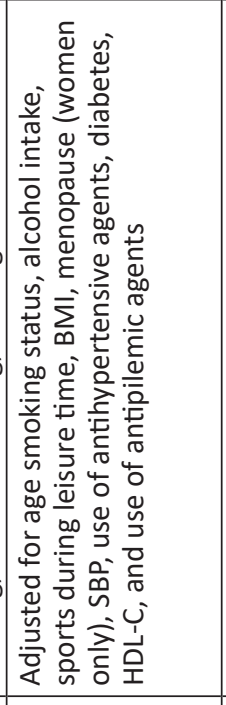 & 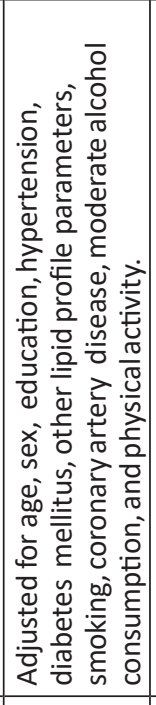 & 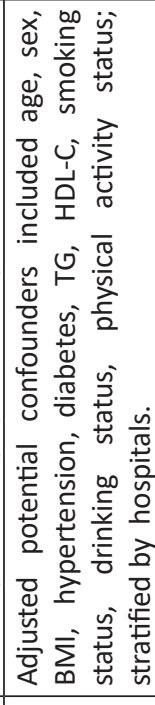 \\
\hline 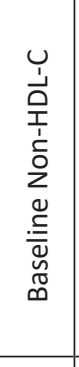 & 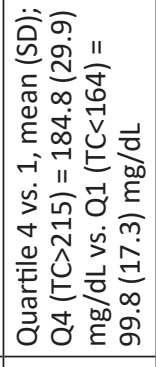 & 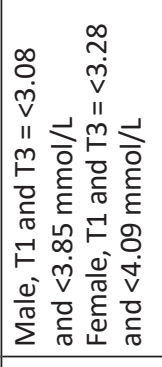 & 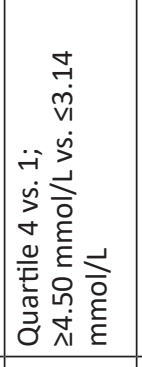 & 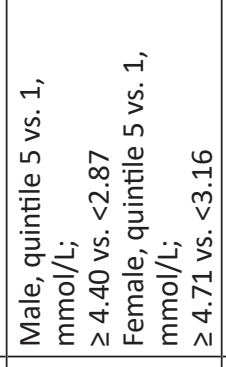 & 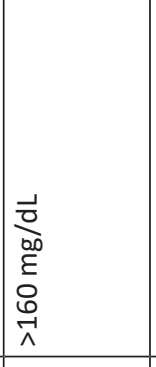 & 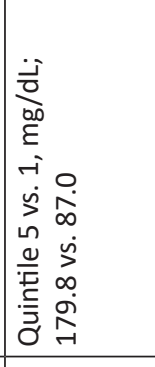 \\
\hline 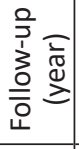 & 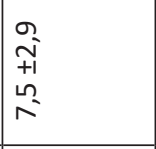 & $\hat{\vec{\sigma}}$ & $\stackrel{\Xi}{\sim}$ & $\stackrel{\leftrightarrow}{\sim}$ & $\stackrel{\stackrel{n}{N}}{\stackrel{2}{2}}$ & $\nabla$ \\
\hline 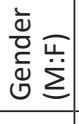 & 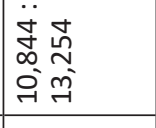 & 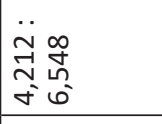 & 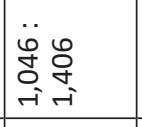 & 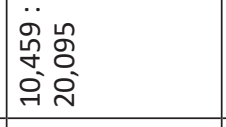 & $\begin{array}{ll}\ddot{i} & 0 \\
0 & 0 \\
0 & 0 \\
-i & -i\end{array}$ & 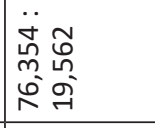 \\
\hline 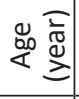 & $\stackrel{\text { n̊ }}{\wedge}$ & $\begin{array}{l}\text { ஸे } \\
\text { ஸे }\end{array}$ & $\stackrel{P}{\wedge}$ & $\begin{array}{l}0 \\
0 \\
0 \\
\dot{q}\end{array}$ & $\underset{\wedge}{\stackrel{\rho}{\wedge}}$ & $\stackrel{\infty}{\wedge}$ \\
\hline $\begin{array}{l}\frac{\tilde{U}}{\omega} \\
\frac{.}{\vec{a}} \\
\vec{\omega}\end{array}$ & $\begin{array}{l}\infty \\
o \\
0 \\
\stackrel{d}{\sim}\end{array}$ & $\begin{array}{l}8 \\
0 \\
0 \\
0 \\
-1\end{array}$ & 帒 & 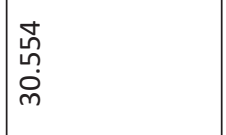 & $\begin{array}{l}\text { g } \\
\text { d } \\
\text { i }\end{array}$ & 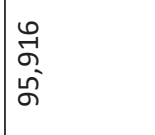 \\
\hline 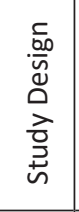 & 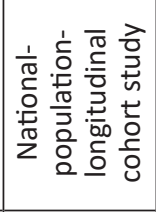 & 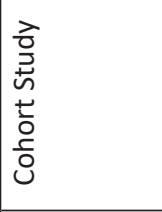 & 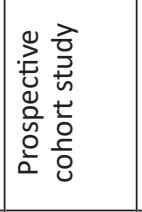 & 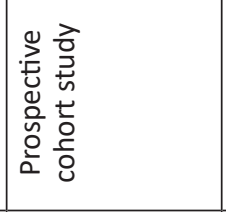 & 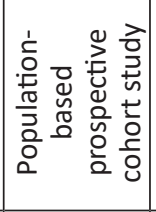 & 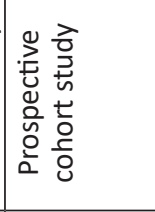 \\
\hline 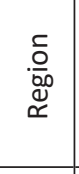 & 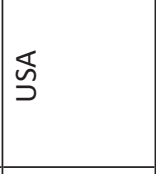 & 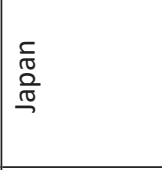 & 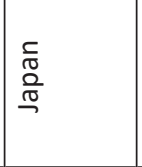 & \begin{tabular}{|l}
$\frac{5}{\pi}$ \\
$\frac{0}{0}$ \\
$\frac{0}{\pi}$
\end{tabular} & 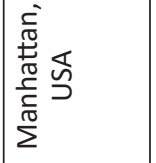 & 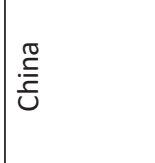 \\
\hline 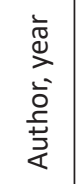 & $\begin{array}{l}0 \\
0 \\
0 \\
N \\
\ddot{\Phi} \\
\tilde{\omega} \\
\frac{\pi}{v}\end{array}$ & 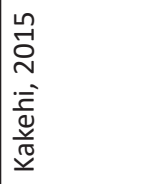 & 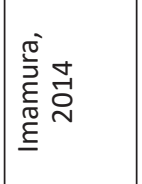 & 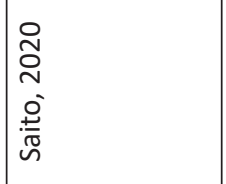 & 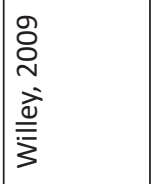 & \begin{tabular}{l}
$m$ \\
$\stackrel{m}{0}$ \\
\multirow{3}{3}{}
\end{tabular} \\
\hline
\end{tabular}


TABLE 2. Key summaries of qualitative appraisal using STROBE statements

\begin{tabular}{|c|c|c|c|c|c|c|c|c|c|c|c|c|c|c|c|c|c|c|c|c|c|c|}
\hline \multirow[b]{2}{*}{ Title } & \multicolumn{22}{|c|}{ STROBE Statement } \\
\hline & 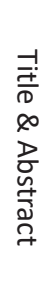 & 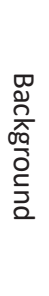 & 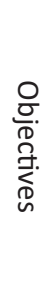 & 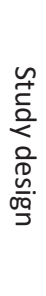 & 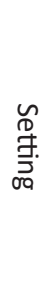 & 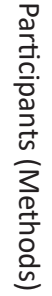 & $\begin{array}{l}\frac{\sigma}{\bar{a}} \\
\frac{0}{0} \\
\frac{\sigma}{D}\end{array}$ & $\begin{array}{l}\text { D } \\
\stackrel{D}{0} \\
\text { w } \\
\text { O } \\
\bar{D} \\
\stackrel{D}{D}\end{array}$ & D. & $\begin{array}{l}\frac{n}{c} \\
\frac{2}{2} \\
\frac{\sim}{N} \\
D\end{array}$ & 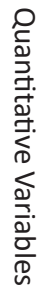 & 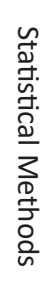 & 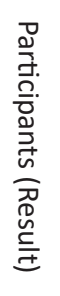 & 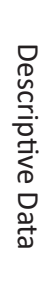 & 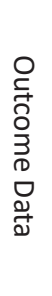 & 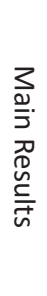 & 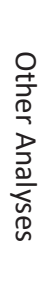 & 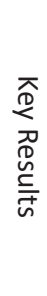 & 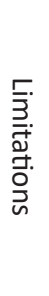 & 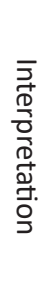 & 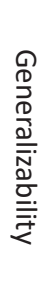 & 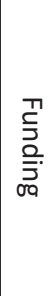 \\
\hline $\begin{array}{c}\text { Glasser, } \\
2016\end{array}$ & & & & & & & & & & & & & & & & & & & & & & \\
\hline $\begin{array}{c}\text { Kakehi, } \\
2015\end{array}$ & & & & & & & & & & & & & & & & & & & & & & \\
\hline $\begin{array}{c}\text { Imamura, } \\
2014\end{array}$ & & & & & & & & & & & & & & & & & & & & & & \\
\hline $\begin{array}{l}\text { Saito, } \\
2020\end{array}$ & & & & & & & & & & & & & & & & & & & & & & \\
\hline $\begin{array}{l}\text { Willey, } \\
2009\end{array}$ & & & & & & & & & & & & & & & & & & & & & & \\
\hline Wu, 2013 & & & & & & & & & & & & & & & & & & & & & & \\
\hline
\end{tabular}

complete $\bigcirc$ less complete $\bigcirc$ incomplete

pertension, diabetes, and obesity among African Americans (2). Another study in the Manhattan, United States, showed different races (6). The races in the study were divided into three, i.e., Hispanic, non-Hispanic blacks, and non-Hispanic whites with the percentage of the Hispanic race was $53.1 \%$. The study showed a correlation, but insignificant, between cholesterol level and the risk of ischemic stroke. Race differences also influenced the study result. A study in Manhattan showed that the Hispanic race had a lower risk compared to the black race, with the incidence of $13 / 1,000$ people per year for the black race and $10 / 1,000$ people per year for the Hispanic race (12).

Other studies conducted in two East Asian countries, i.e., Japan and China (7-10), also provided different results. This can be affected by the correlation of several risk factors in various East Asian countries. China and Mongolia had an increase in stroke incidents from 1990 to 2010, followed by an increase in hypertension prevalence. Meanwhile, there was a decrease in stroke incidence in Japan and Korea, with a decreased prevalence of hypertension. The global estimate for diabetes prevalence in 2013 by the International Diabetes Federation in China was $9.0 \%$, while Ja- pan was $5.1 \%$, similar to the United States, with $9.2 \%$ and Canada with $7.9 \%$. More than $40 \%$ of diabetic patients live in East Asia. In general, the population in East Asia has lower total serum cholesterol compared to western countries. Japan has been one of the countries with the lowest total serum cholesterol. However, it increased from 19581986. China had a higher increase in total serum cholesterol than Japan for female individuals. Other than that, China placed first with 301 million smokers, which consisted of $28.1 \%$ adults in 2010 (13). In China, the risk of stroke is related to an unhealthy lifestyle, such as smoking, obesity, alcohol consumption, low physical activity, and related to dietary intake (14).

A previous meta-analysis revealed that individuals with the highest serum level of non-HDL-C had a $28 \%$ increase of total stroke risk. Subgroup analysis showed that total stroke risk was stronger in male participants compared to female participants. The study also stated that increased serum non-HDL-C was independently associated with a higher risk of ischemic stroke (15). However, the association with hemorrhagic stroke was unclear. This was in line with the results from two studies, which indicated a significant correlation between increased on-HDL-C and the risk of stroke ischem- 
ic $(5,10)$. Meanwhile, the result of these meta-analyses disagreed with the results of the other four studies (6-9).

There were several limitations to this systematic review. First, the threshold level of non-HDL-C still varied between all studies. Second, there was a lack of adjustments to the use of cholesterol-lowering drugs and other confounding factors that may affect several studies. Third, in several studies, the female to male ratio was too large, thus cannot be used to represent all populations.

\section{CONCLUSIONS}

This systematic review aimed to determine the correlation between high serum level of nonHDL-C and the risk of ischemic stroke, with the

Conflict of interest: none declared

Financial support: none declared

\section{REFERENCES}

1. Johnson W, Oyere O, Mayowa O, Sonal S. Stroke: a global response is needed. Bulletin of the World Health Organization. 2016;94:634-634A.

2. Boehme AK, Charles E, Mitchell S. Stroke Risk Factors, Genetics, and Prevention. Circ Res. 2017;120:472-495.

3. Siddeswari R, Manohar S, Sudarsi B, et al. Pattern of dyslipidemia in ischemic stroke. J Med Allied Sci. 2015;5(2):26-29.

4. Chowdhury S, Jyothi R, Soumik G. The Importance of Non High Density Lipoprotein Cholesterol in Dyslipidaemia Management. J Diabetes Metab. 2015;6(11):2-5.

5. Glasser SP, Aleena M, Maciej B, George H. What Is The Association Of Lipid Levels And Incident Stroke?. Int J Cardiol. 2016; 220:890-894.

6. Willey JZ, Qiang Xu, Bernadette BA et al. Lipid Profile Components and Risk of Ischemic Stroke: The Northern Manhattan Study (NOMAS). Arch Neurol. 2009;66(11):1400-1406.

7. Kakehi E, Kazuhiko K, Shizukiyo I, et al. Serum Non-High- Density Lipoprotein Cholesterol Levels and the Incidence of Ischemic Stroke in a Japanese Population: The Jichi Medical School Cohort Study. Asia-Pacific Journal of Public Health. 2015;27(2):535-543.

8. Imamura $\mathrm{T}$, Yasufumi $\mathrm{D}$, Toshiharu $\mathrm{N}$, et al. Non-high-density lipoprotein cholesterol and the development of coronary heart disease and stroke subtypes in a general Japanese population: The Hisayama Study. Atherosclerosis. 2014;233:343-348. outcome of HR and 95\% CI. Based on the six studies reviewed, there were conflicting results. Two studies with a larger total population stated a significant correlation between high serum level of non-HDL-C and the risk of ischemic stroke. Meanwhile, the other four studies stated otherwise.

In the future, it is expected that many studies specifically investigate the correlation between the non-HDL-C level and the risk of ischemic stroke. Further studies are expected to consider confounding factors, such as the use of lipid-lowering drugs and other risk-related factors that may influence the study results.

\section{Acknowledgement}

Each named author has substantially contributed to this article.
9. Saito I, Kazumasa Y, Yoshihiro K, et al. Non-High-Density Lipoprotein Cholesterol and Risk of Stroke Subtypes and Coronary Heart Disease, The Japan Public Health Center-Based Prospective (JPHC) Study. J Atheroscler Thromb. 2020;27:363-374.

10. Wu J, Shengyun C, Yong Z et al. Non-High-Density Lipoprotein Cholesterol on the Risks of Stroke: A Result from the Kailuan Study. Cholesterol and Stroke. 2013;8(9):1-8.

11. Greenland S, Stephen JS, Kenneth JR et al. Statistical Tests, P Values, Confidence Intervals, And Power: A Guide To Misinterpretations. Eur J Epidemiol. 2016:3:337-350.

12. Gardener H, Ralph LS, Tatjana R, et al. Race and Ethnic Disparities in Stroke Incidence in the Northern Manhattan Study. Stroke. 2020;51:1064-1069.

13. Kim YD, Yo HJ, Gustavo S. Traditional Risk Factors for Stroke in East Asia. Journal of Stroke. 2016;18(3):273-285.

14. Li Y, Yuna He, Jianqiang L, et al. Dietary Patterns Are Associated with Stroke in Chinese Adults. The Journal of Nutrition. 2011; 141:1834-1839.

15. Wang P, Jian Sun, Tao Xie, dan Sujun LV. Serum non-high- density lipoprotein cholesterol and risk of stroke in the general population: a meta-analysis. Int J Clin Exp Med. 2018;11(3):1467-1474. 